\title{
Chylothorax after Blunt Chest Trauma: A Case Report
}

\author{
Pawit Sriprasit, M.D. ${ }^{1}$, Osaree Akaraborworn, M.D., M.Sc. ${ }^{2}$ \\ ${ }^{1}$ Department of Surgery and ${ }^{2}$ Division of Trauma Surgery, Department of Surgery, \\ Faculty of Medicine, Prince of Songkla University
}

Traumatic chylothorax after blunt chest trauma alone is considered rare. Our patient was a 27-year-old female who was in a motorcycle accident and sustained blunt thoracic and traumatic thoracic aortic injuries with T1-T2 vertebral subluxation. She underwent thoracic endovascular aortic repair from T4 to T9 without any thoracic or spinal surgery. On postoperative day 7, the drainage from her left chest turned into a milky-white fluid indicative of chyle leakage. The patient was treated conservatively for 2 weeks and then the chest drain was safely removed. The results show that traumatic chylothorax can be successfully managed with conservative treatment.

Key words: 1. Chylothorax

2. Chest injuries

3. Thoracic injuries

\section{Case report}

Traumatic chylothorax from blunt injury of the chest is considered to be rare. Recent studies suggest that it can result from an injury to the thoracic duct, which then causes chyle leakage. We report here a case of diagnosed blunt traumatic chylothorax that responded successfully to conservative management.

Our patient was a 27-year-old female who was injured in a motorcycle accident. She was referred from a nearby hospital to the specialized vascular center to receive care for a thoracic aortic injury. Initially, she had a subarachnoid hemorrhage, bilateral traumatic hemothoraces, posterior subluxation of T1-T2, neurogenic shock, and multiple fractures of the extremities. She underwent thoracic endovascular aortic repair (TEVAR) on the same day she arrived at the center. After surgery, her clinical condition improved, and on postoperative day 3, she was transferred from the surgical intensive care unit to the general ward. Oral nutrition was started on the fifth day, upon observation that her chest drainage became serosanguineous in color and clear, having decreased from $100 \mathrm{~mL} /$ day on the left side and 300 $\mathrm{mL} /$ day on the right side on the first day. On postoperative day 6, she developed a high-grade fever. The investigation showed positive results for pneumonia, and empirical antibiotics were prescribed. A chest X-ray taken at this time showed improved drainage of the pleural effusion (Fig. 1). On postoperative day 7 , the drainage from her left chest turned milky-white, and a specimen was obtained for analysis (Fig. 2). The fluid analysis indicated a glucose level of $103 \mathrm{mg} / \mathrm{dL}$; a protein concentration of $2.2 \mathrm{~g} / \mathrm{dL}$; a lactate dehydrogenase level of 1,196 U/L; a white blood cell count of 810 cells $/ \mathrm{mm}^{3} ; 62 \%$ mononuclear and $25 \%$ polymorphonuclear cells; a red blood cell count of 32,250 cells $/ \mathrm{mm}^{3}$; a fluid triglyceride level of $1,317 \mathrm{mg} / \mathrm{dL}$; and a negative fluid aerobic culture. She was diagnosed with chylothorax

Received: November 10, 2016, Revised: April 20, 2017, Accepted: May 9, 2017, Published online: October 5, 2017

Corresponding author: Pawit Sriprasit, Department of Surgery, Faculty of Medicine, Prince of Songkla University, Hat Yai, Songkhla, Thailand 90110

(Tel) 66-896580990 (Fax) 66-74429384 (E-mail) pawit.sriprasit@yahoo.com

( $)$ The Korean Society for Thoracic and Cardiovascular Surgery. 2017. All right reserved.

@i) This is an open access article distributed under the terms of the Creative Commons Attribution Non-Commercial License (http://creativecommons.org/ licenses/by-nc/4.0) which permits unrestricted non-commercial use, distribution, and reproduction in any medium, provided the original work is properly cited. 


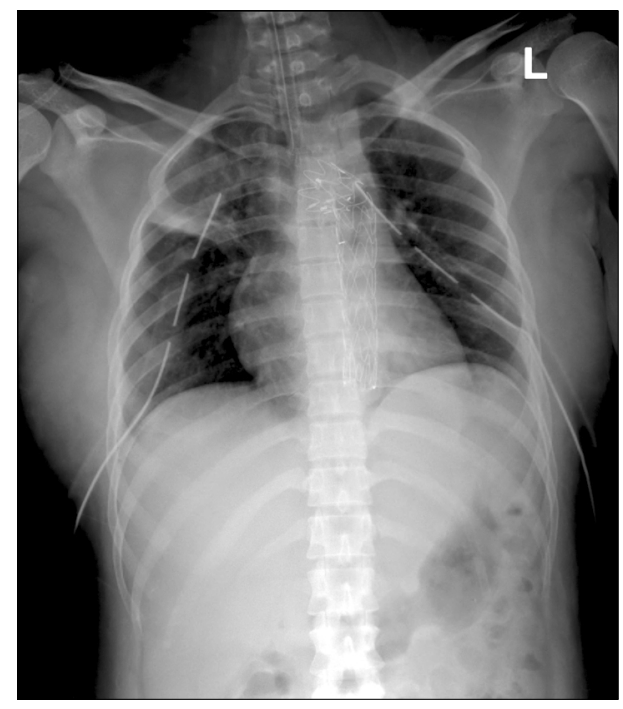

Fig. 1. Chest X-ray of the patient, 7th day post operation.

on the left side through a biochemical analysis, which was sufficient to support the definitive diagnosis without nuclear imaging, and was initially treated with nil per os (NPO) and a peripheral parenteral nutritional infusion. After NPO, her left chest drainage gradually decreased to $80-100 \mathrm{~mL} /$ day and became clear on day 5 after the diagnosis. On the ninth day after the diagnosis of chylothorax, lymphoscintigraphy was performed using $1.3 \mathrm{mCi}$ of 99mTc-dextran injected via the left foot to confirm that the patient was healing and to rule out any major injuries. Dynamic images were taken every 10 seconds for 1 hour, and the results showed no evidence of lymphatic duct leakage. An oral diet was planned to be started at this time, but she underwent C7-T3 instrumental fixation by an orthopedist on the following day, so it was postponed until day 12 after the chylothorax was diagnosed. The left chest drainage was reanalyzed, and it showed a fluid triglyceride level of $37 \mathrm{mg} / \mathrm{dL}$. The chest drain was safely removed on the 17 th day post TEVAR.

\section{Discussion}

Traumatic chylothoraces can be iatrogenic, secondary to operative procedures, or caused by percutaneous thoracic procedures that result in trauma to the lymphatic drainage of the left subclavian vein. Nonsurgical blunt or penetrating trauma can also injure the lymphatic system, but it is quite uncommon

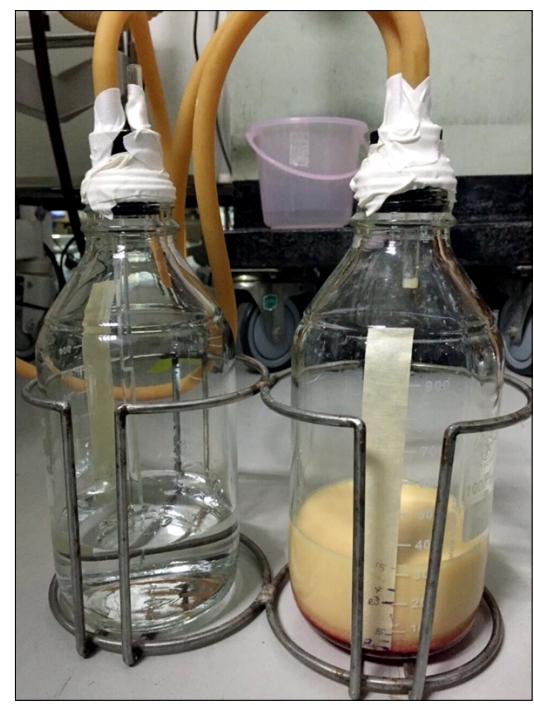

Fig. 2. Fluid drained from the chylothorax, 7th day post operation.

[1]. The longest onset that has been reported is 20 years after the injury. It was hypothesized by Milano et al. [2] that the chyle and lymph had collected slowly in the posterior mediastinum until it leaked into the pleural space [3].

A previous study hypothesized that blunt injuries cause hyperextension of the spine, which cause a disruption of the thoracic duct. Normally, the thoracic duct travels from the aortic hiatus at the 10th thoracic vertebra on the right, ascends up and crosses to the left at the fifth or sixth level of the thoracic vertebra, and travels into the posterior mediastinum before draining into the subclavian vein. This describes about $65 \%$ of the population $[1,4,5]$. Possible duct injury from central venous puncture was reported to have resulted from the insertion of a central line [6]. The possibility that this case was caused by the TEVAR is low, because TEVAR only exerts intra-arterial mechanical effects, and there are no previous reports of TEVAR-related traumatic chylothorax. In our case, the patient's chylothorax possibly resulted from injuries at the aortic arch and at the second thoracic vertebra corresponding to the left-sided thoracic lymph duct. In addition to the spinal injury, other rarely reported causes of chylothorax are seat-belt-related injuries and an increase in thoracic or abdominal pressure [1].

The pleural fluid should be rich in lipids and protein, but it does not always appear milky-white, because it can mix with other components, as in cases 
of traumatic hemothorax. Fifty percent of patients with chylothorax have bloody, yellow, or green turbid fluid [1,3,7]. Moreover, a white color can mimic thoracic empyema, underscoring the importance of a clinical differential diagnosis. A laboratory examination of the chylothorax should include a quantitative analysis of the fluid chemistry. Pleural effusions with triglyceride values estimated at more than 110 $\mathrm{mg} / \mathrm{dL}$ have a $99 \%$ probability of being indicative of chylothorax. Values that are less than $50 \mathrm{mg} / \mathrm{dL}$ have a less than $5 \%$ probability of being indicative of chylothorax. For values between 50 and $110 \mathrm{mg} / \mathrm{dL}$, the diagnosis is made by confirming the presence of chylomicrons [3,5,8]. Our patient had a negative fluid aerobic culture and a fluid triglyceride level of 1,317 $\mathrm{mg} / \mathrm{dL}$, which indicated chylothorax.

Several techniques are used to confirm the location of chyle leaks. Along with a lipid profile of the fluid, chylomicron staining can be used for a definitive diagnosis. Historically, bipedal lymphangiography (LG) was an invasive technique for lymphatic mapping. However, a high-fat meal mixed with methylene blue has helped in cases involving patients who were allowed food $[1,8]$. There are 2 techniques of LG: pedal and intranodal. Both techniques can successfully identify the leakage site in between $64 \%$ and $86 \%$ of patients with chylothorax and chylous ascites. In our case, the diagnosis of chylothorax was based on the biochemical analysis, so a lymphatic radionuclear scan was not performed at the time. It was later performed only after a period of conservative treatment. The scan showed no evidence of leakage, indicating that the lesion had probably healed.

The contents of the thoracic duct include chyle and lymph from the gut, liver, and lower extremities. Chyle contains chylomicrons, triglycerides, fat-soluble vitamins, cholesterol, and electrolytes similar to serum. Chyle leakage into the pleural cavity can lead to a loss of lipids, electrolytes, water, and proteins $[1,3,8]$. Management of chylothorax depends on the etiology, volume, and situation. Approximately 1,500$2,000 \mathrm{~mL}$ of lymph is produced every day. Common conservative management of chylothorax involves chest drainage, NPO, intravenous fluid resuscitation, and nutritional supplements to promote fistula closure through lung expansion. Patients are kept on NPO status until the chest drainage volume decreases. This method achieves an $88 \%$ success rate within 1-4 weeks. In cases where conservative management fails after 2 weeks or the amount of chyle leakage is more than $1 \mathrm{~L}$ per day for 5 consecutive days or $1.5 \mathrm{~L}$ per day in adults, surgical management options are considered. These include (1) ligation of the thoracic duct at the leakage site via thoracotomy or (2) video-assisted thoracoscopic surgery $[1,6,7]$. Both techniques involve the en masse ligature of the tissue encountered in the supradiaphragmatic region between the descending thoracic aorta and the azygos vein using clips or nonabsorbable sutures. Duct ligation in the supradiaphragmatic region offers a $90 \%$ success rate [6]. Our patient underwent conservative treatment. On the ninth day after the chylothorax diagnosis, success was confirmed by lymphoscintigraphy. After that, she was able to transition to oral nutrition without complications.

In summary, blunt traumatic chylothorax can occur in cases of torso trauma. A definitive diagnosis should be made through laboratory testing prior to management. Most patients with these conditions are successfully managed with conservative treatment. If there is no improvement after 2 weeks, surgical management should be considered.

\section{Conflict of interest}

No potential conflicts of interest relevant to this article are reported.

\section{Acknowledgments}

This research was funded by Faculty of Medicine, Prince of Songkla University, Thailand.

\section{References}

1. Seitelman E, Arellano JJ, Takabe K, Barrett L, Faust G, Angus LD. Chylothorax after blunt trauma. J Thorac Dis 2012;4:327-30.

2. Milano S, Maroldi R, Vezzoli G, Bozzola G, Battaglia G, Mombelloni G. Chylothorax after blunt chest trauma: an unusual case with a long latent period. Thorac Cardiovasc Surg 1994;42:187-90.

3. Grant PW, Brown SW. Traumatic chylothorax: a case report. Aust N Z J Surg 1991;61:798-800.

4. Laaveg SJ, Sprague BL. Traumatic chylothorax, a complication of fracture-dislocation of the spine: a case report. J Bone Joint Surg Am 1978;60:708-9. 
5. Platz A, Simmen HP, Heinzelmann M, Kohler A, Trentz O. Chylothorax after blunt thoracic trauma. Chirurg 1995; 66:127-30.

6. Pillay TG, Singh B. A review of traumatic chylothorax. Injury 2016;47:545-50.

7. Ikonomidis JS, Boulanger BR, Brenneman FD. Chylothorax after blunt chest trauma: a report of 2 cases. Can J Surg 1997;40:135-8.

8. Apostolakis E, Akinosoglou K, Koletsis E, Dougenis D. Traumatic chylothorax following blunt thoracic trauma: two conservatively treated cases. J Card Surg 2009;24: 220-2. 